\title{
Minimally invasive management of pancreatic pseudocysts
}

\author{
Audrius Šileikis', Augustas Beiša', Elena Zdanytè2, Saulius Jurevičius', Kęstutis Strupas ${ }^{1}$ \\ ${ }^{1}$ Clinic of Gastroenterology, Nephrology and Surgery, Medical Faculty, Vilnius University, Lithuania \\ ${ }^{2}$ Centre of Abdominal Surgery, Hospital Santariškių Klinikos, Vilnius University, Lithuania
}

Videosurgery Miniinv 2013; 8 (3): 211-215

DOI: $10.5114 /$ wiitm.2011.33809

\begin{abstract}
Introduction: The laparoscopic and endoscopic approaches to internal drainage of pancreatic pseudocysts (PP) are the current minimally invasive management options. Indications, and early and late results of endoscopic and laparoscopic approaches are being discussed.

Aim: To present experience in treatment of PP by laparoscopic pseudocystogastrostomy (LPGS) and endoscopic pseudocystogastrostomy (EPGS) and to compare results, feasibility and safety.

Material and methods: Thirty patients underwent surgical intervention: 18 patients - LPGS (group I), 12 - EPGS (group II). Groups were compared by age, gender, pancreatic pseudocysts's age, diameter and localization, as well as intraoperative, early and late postoperative complications.

Results: Gender distribution, group I: 14 (77.8\%) men and 4 (22.2\%) women, group II: 4 (33.3\%) men and 8 (66.7\%) women, $p=0.02$. Average cyst diameter: group I $149.9 \pm 52.1 \mathrm{~mm}$, group $/ I-119 \pm 37.9 \mathrm{~mm}, p=0.07$. Average time between diagnosis and operation performance: group I - 12 (3-60) months, group II - 8 (2-36) months, $p=0.19$. Neither in group I nor in group II did intraoperative complications occur. Early postoperative complications were divided into minor and major. Early minor complications: group I - 2 (11.1\%), group II - 0, $p=0.5$. Early major complications: group I - 0, group II - 2 (16.7\%), $p=0.15$. Late postoperative complications: group I - 0, group II -1 (8.3\%), $p=0.4$. In group I there was no case, whereas in II group there was 1 (8.3\%) case of recidivation, $p=0.4$.

Conclusions: For selected patients both minimally invasive methods are equally safe an effective. For comprehensive evaluation of methods prospective trials are needed.
\end{abstract}

Key words: pancreatic pseudocysts, laparoscopic pseudocystogastrostomy, endoscopic pseudocystogastrostomy.

\section{Introduction}

Laparoscopic and minimally invasive surgery methods have become the daily routine in our operating rooms. Pancreatic surgery is not an exception. The laparoscopic and endoscopic approaches to internal drainage of pancreatic pseudocysts (PP) are the current minimally invasive management options $[1,2]$. Indications, and early and late results of endoscopic and laparoscopic approaches are being discussed.

\section{Aim}

The purpose of this article is to present our experience in treatment of PP by laparoscopic and endoscopic pseudocystogastrostomy, to compare results and to evaluate feasibility and safety, and to discuss advantages and disadvantages of both methods.

\section{Material and methods}

We performed a retrospective analysis of laparoscopic pseudocystogastrostomy (LPGS) and endo- 
scopic pseudocystogastrostomy (EPGS) results. Between 2008 and 2011 there were 18 patients who underwent LPGS (group I), and 12 patients who underwent EPGS (group II). For all patients presence of PP was proved by one of these methods: computerized tomography $(\mathrm{CT})$ and magnetic resonance imaging (MRI) with endoscopic ultrasound (EUS) and fine needle aspiration (FNA) for cytological, biochemical ( $\alpha$-amylases, carcinoembryonic antigen (CEA)) examination. Patients with CEA $<192$, high $\alpha$-amylases and no atypical cells were selected for minimally invasive surgery procedures. During the operation PP fluid was sampled for cytological, biochemical $(\alpha$-amylases, CEA), and microbiological examination. Because EPGS is technically impossible for bigger (> $18 \mathrm{~cm}$ diameter), multilocular or PP containing ne-

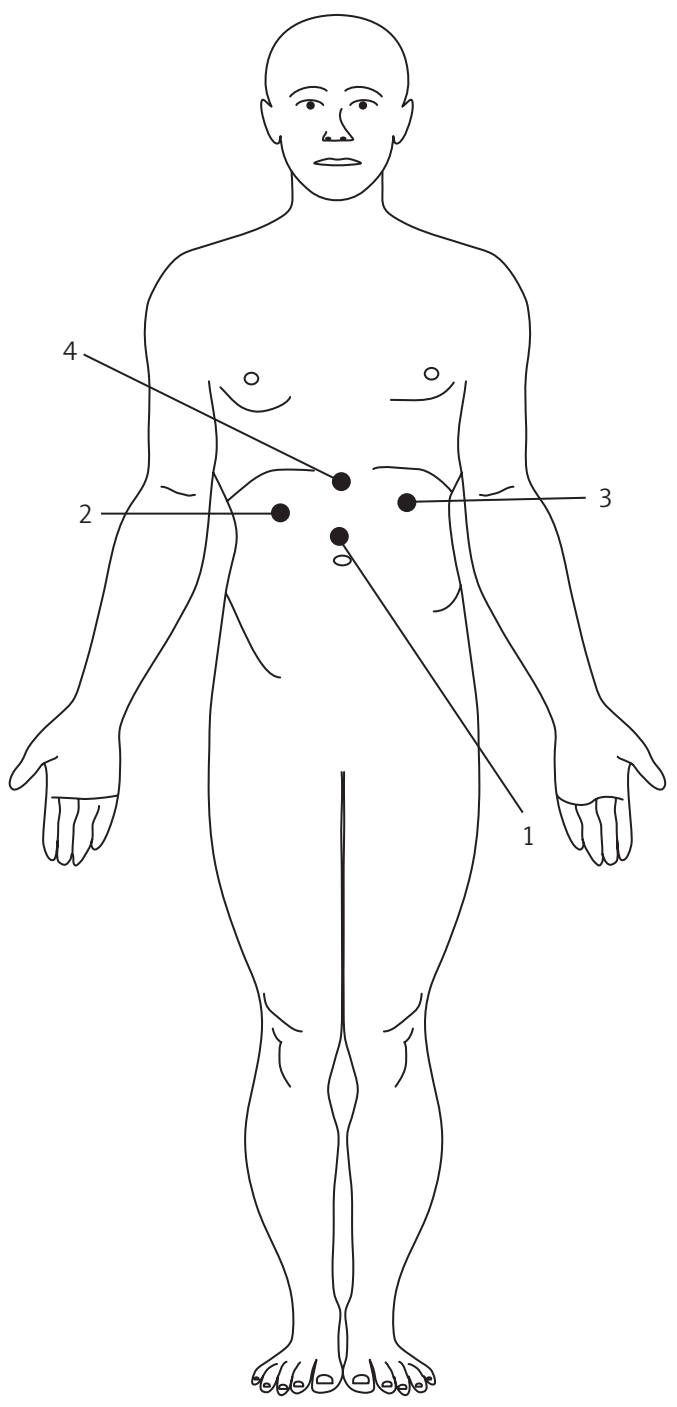

Figure 1. Positioning of the trocars crotic debris they were managed by LPGS. Intraoperative, early and late postoperative complications were evaluated and compared.

\section{Technique of laparoscopic pseudocystogastrostomy (Video)}

This technique was described previously by Šileikis et al. [3]. The patient was placed on the operating table in the supine semi-lateral right side up position with the surgeon on the patient's right and the first assistant on the patient's left, and the camera man behind the surgeon on the patient's right. Positioning of trocars (Figure 1): After general anesthesia a $10 \mathrm{~mm}$ port (1) was placed supraumbilically for laparoscopy, and two $10 \mathrm{~mm}$ ports were placed in typical positions: the second (2) to the right from the point where medial and superior thirds of the distance between the umbilicus and processus xyphoideus intersects; the third (3) subcostally, in the left anterior axillary line; and the fourth (4), a $10 \mathrm{~mm}$ port, was introduced in the middle line between the umbilicus and processus xyphoideus (Figure 1). The abdominal cavity was inspected through the umbilical trocar with a 30-degree-angle Olympus laparoscope. To confirm localization of PP and its relation to the stomach a laparoscopic ultrasound (LUS) instrument was inserted through the left trocar. After an Ultracision scalpel was introduced through the left trocar and a Babcock forceps through the right, gastrotomy of the anterior wall was performed at the superior third of the corpus of the stomach using the coagulation mode of the scalpel. Under LUS control the laparoscopic needle was introduced to confirm the localization of PP and to sample the fluid. To support the anterior wall of the stomach, a needle was introduced percutaneously and the anterior wall of the stomach was sutured with the abdominal wall. Then the Ultracision scalpel was used to create a cystogastrostomy between the posterior wall of the stomach and the adherent anterior wall of the PP. Anastomosis made between the dorsal wall of the stomach and PP should be 2-4 cm. After necrosectomy and aspiration of content the cavity of the PP was inspected. Electrocautery diathermy and titanium clips were used for hemostasis (if needed). The anterior gastrotomy was closed by single 3/0 absorbed vicryl sutures. For suture quality control methylene blue can be injected through a nasogastric tube. If needed, additional sutures can be made. 
Table I. Evaluation of LPGS and EPGS results

\begin{tabular}{|lccc|}
\hline Parameter & Group I (LPGS) $(n=18)$ & Group II (EPGS) $(n=12)$ & Value of $p$ \\
\hline Average age of patients [years] & $40 \pm 19$ & $45 \pm 8.7$ & 0.34 (T-test) \\
\hline Male/female & $14(77.8 \%) / 4(22.2 \%)$ & $4(33.3 \%) / 8(66.7 \%)$ & 0.02 (Fisher) \\
\hline Acute pancreatitis & $16(88.9 \%)$ & $8(66.7 \%)$ & 0.18 \\
(Fisher)
\end{tabular}

\section{Technique of endoscopic pseudocystogastrostomy}

Intervention was performed after intravenous sedation. Once the bulge is located, its apex is identified by EUS and FNA. After needle puncture of the PP, fluid content is aspirated for examination and a guidewire is inserted, along which an incision is made using either a diathermic coagulation probe or a needle-knife papillotome. Once access has been achieved, a double pigtailed catheter is passed into the PP over the wire.

\section{Results}

The total number of adult patients was 30 . Table I summarizes the comparison of groups. Out of the total number, the majority of patients (60\%) underwent an LPGS (group I), the others EPGS (group II). In group I, $77.8 \%$ of patients were men, whereas in group II the dominating gender was women (66.7\%), $p=0.02$. There was no significant age difference between groups I and II, $p=0.34$. In both groups the majority of patients were after acute necrotizing pancreatitis: group I - $88.9 \%$ of patients, group II $66.7 \%, p=0.18$. Most PP were diagnosed by CT scan: in group | $-88.9 \%$ of cases, in group $\mid$ I $-83.3 \%$. Greater average cyst diameter (the biggest dimen- sion) was found in group I - $149.9 \pm 52.1 \mathrm{~mm}$, but the difference was not significant, $p=0.07$. In group I average thickness of the PP wall was $5.5 \pm 1.1 \mathrm{~mm}$. Average time between diagnosis and operation performance was 12 (3-60) months in group I and 8 (2-36) months in group II, $p=0.19$. Necrotic debris during the operation was found in 16 of our patients (88.9\%) in group I. In both cases the most common location of PP was in the body and tail of the pancreas $-33.3 \%$ in group I and $66.6 \%$ in group II, $p=0.13$. After examination of a fluid sample, infection was diagnosed in $6(33.3 \%)$ patients in group I and none in group II, $p=0.06$. There were no intraoperative complications, no conversions, and no deaths either in group I or in group II. Average time of the operation in group I was $138 \pm 32.1$ min. Early postoperative complications were divided into two groups: minor and major. In group I, 11.1\% of patients had early minor complications (postoperative hemorrhage which required endoscopic hemostasis and hemotransfusion), but no major ones, whereas in group II, $16.7 \%$ of patients had some major complications (peritonitis and suppuration of PP, open surgical interventions were needed). Postoperative stay in group II was shorter at 6 (3-15) days, but it did not have a significant difference from group I, $p=0.17$. For $8.3 \%$ of patients in group II late postoperative 
complications (later than 21 days after intervention) occurred (infection of PP content, additional pseudocystogastrostomy was needed). During 12 months of the follow-up period there was no recidivation in group I, whereas in group II recurrence of PP occurred in $8.3 \%$ of patients.

\section{Discussion}

The first documented description of a PP can be ascribed to Morgagni in 1761 [4]. The first pseudocystogastrostomy was performed in 1921 [5]. Currently laparoscopic and endoscopic methods have become the main options of choice in PP treatment. Endoscopic pseudocystogastrostomy was performed in 1985 by Kozarek. Laparoscopic pseudocystogastrostomy was performed in 1991 [3].

A PP is a complex entity with multiple characteristics, each of which may dictate one method over another [4]. Endoscopic intervention is only feasible when a favorable anatomical situation is present such as a common wall between the PP and the stomach/duodenum, absence of varices, maximum distance between PP and gastric wall of less than $1 \mathrm{~cm}$ for transmural drainage, or an existing communication between the PP and the pancreatic ductal system for transpapillary drainage [6]. Findings have shown endoscopic drainage to be unsuitable for patients with PP in the tail of the pancreas due to difficulty in achieving access and patients with a wall thickness of $1 \mathrm{~cm}$ or greater [1]. Aljarabah et al. hold that endoscopic drainage is more suitable for chronic PP within the head and body of the gland, whereas acute PP, particularly those that complicate necrotizing pancreatitis, are best managed with laparoscopic surgery [7]. Sheng et al. state that endoscopic drainage is associated with a high rate of technical failure, PP recurrence, infection, bleeding, stent blockage, and inadequate drainage [7]. In our data out of 12 patients who underwent EPGS 2 had PP infection in the early postoperative period and one later. Recurrence with the endoscopic approach may be explained in part by inadequacy of the size of the stoma created endoscopically, with subsequent blockage of stents, particularly in patients with acute rather than chronic PP $[1,8]$. In our data, we had 1 case of recidivation during 12 months of follow-up. The endoscopists are less equipped to deal with bleeding than the laparoscopic surgeon, who has a wider access to a range of options such as suturing, the ultrasonically activated scalpel, vascular endostaplers, endoclips, and hemostatic thrombin [1].

As experience with minimally invasive techniques has grown, laparoscopy is being increasingly used for the creation of pseudocystogastrostomies [9]. Laparoscopic drainage of mature PP is minimally invasive and offers definitive drainage. With the availability of advanced imaging systems and cameras, better hemostatic equipment and excellent suturing skills, most PP can be approached and managed by a laparoscopic approach [10]. Laparoscopic procedures for PP include pancreatic pseudocystogastrostomy, pseudocystoduodenostomy, and pseudocystojejunostomy. Out of all transgastric approaches, anterior gastrotomy is associated with better exposure and shorter operative time [11, 12]. Laparoscopy seems to have a distinct advantage over endoscopic drainage in the case of PP that contain significant debris or necrosis because of the larger size of the stoma created. Pancreatic necrosis is a common cause of failure with the endoscopic transmural approach where stents are used as necrotic debris may lead to stent occlusion with subsequent secondary infection of the PP and necrotic tissue, sepsis, and recurrence. Surgery, either laparoscopic or conventional, may be preferable when adjunctive procedures such as cholecystectomy need to be performed. In fact, in one series more than $50 \%$ of patients had gallstone pancreatitis necessitating laparoscopic or open cholecystectomy [4]. Although some advocate routine suturing of the pseudocystogastrostomy to prevent postoperative bleeding, others have not found this to be routinely necessary and have not encountered postoperative bleeding $[11,13]$. We report 2 cases from 18 of early postoperative bleeding from pseudocystogastrostomy. Routine suturing of pseudocystogastrostomy was not used. In a systematic review of the literature, laparoscopic drainage was associated with low morbidity, rapid recovery, and recurrence rates comparable to those reported by open surgery $[11,14]$. During 12-month follow-up we did not have any case of cyst recidivation. Although laparoscopic management has been reported with very encouraging results, long-term follow-up has still to show equivalence to open surgery [6].

\section{Conclusions}

Both minimally invasive methods are equally safe and effective for selected patients. Laparoscopic 
pseudocystogastrostomy, because of the technical limits of EPGS, should be chosen for multilocular and PP with necrotic debris or PP localized in the tail of the pancreas. Advantages of LPGS are: wider stoma (for adequate drainage), possibility of necrosectomy, low postoperative infection and recidivation rates. Endoscopic pseudocystogastrostomy should be chosen for patients with smaller, non-complicated PP. Advantages of EPGS are: less invasive compared to LPGS and shorter postoperative stay. Although LPGS results are encouraging, for more comprehensive evaluation prospective trials are needed.

\section{References}

1. Aljarabah M, Ammori BJ. Laparoscopic and endoscopic approaches for drainage of pancreatic pseudocysts: a systematic review of published series. Surg Endosc 2007; 21: 1936-44.

2. Makarewicz W, Kaska $Ł$, Stefaniak T, et al. Videoscopic procedures in gastrointestinal and endocrine surgery. Videosurgery Miniinv 2006; 2: 87-93.

3. Šileikis A, Skrebūnas A, Beiša V, et al. Laparoscopic pseudocystogastrostomy for the treatment of pancreatic pseudocyst: clinical case and review of the literature. Medicinos teorija ir praktika 2010; 3: 287-92.

4. Gumaste VV, Aron J. Pseudocyst management endoscopic: drainage and other emerging techniques. J Clin Gastroenterol 2010; 44: 326-31.

5. Jedlica R. Eine neue Operationsmethode der Pancreascysten (Pancreatogastrostomie). Zentralbl Chir 1923; 50: 132.

6. Aghdassi A, Mayerle J, Kraft M, et al. Diagnosis and treatment of pancreatic pseudocysts in chronic pancreatitis. Pancreas 2008; 36: 105-12.

7. Sheng QS, Chen DZ, Lang R, et al. Laparoscopic cystogastrostomy for the treatment of pancreatic pseudocysts: a case report. World I Gastroenterol 2008; 14: 4841-3.

8. Behrns KE, Ben-David K. Surgical therapy of pancreatic pseudocysts. J Gastrointest Surg 2008; 12: 2231-9.

9. Gumbs AA. Video: laparoscopic anterior cystogastrostomy. Surg Laparosc Endosc Percutan Tech 2010; 20: e97-8.

10. Palanivelu C, Senthilkumar K, Madhankumar MV, et al. Management of pancreatic pseudocyst in the era of laparoscopic surgery - experience from a tertiary centre. Surg Endosc 2007; 21: 2262-7.

11. Hamza N, Ammori BJ. Laparoscopic drainage of pancreatic pseudocysts: a methodological approach. J Gastrointest Surg 2010; 14: 148-55.

12. Michalik M, Bobowicz M, Lech P, et al. Distal pancreatic resection via laparo-endoscopic single site surgery - development of the technique. Videosurgery Miniinv 2010; 5: 142-5.

13. Korlacki W, Dzielicki J, Grabowski A. Laparoscopic distal pancreatectomy in children with solid pseudopapillary tumour (Frantz tumour) - a report of two cases and review of the literature. Videosurgery Miniinv 2010; 5: 65-9.
14. Kurnicki J, Świątkiewicz J, Wrzesińska N, et al. Laparoscopic treatment of a huge mesenteric pseudocyst - case report. Videosurgery Miniinv 2011; 6: 167-72.

Received: 30.07.2012, accepted: 8.02.2013. 